\title{
Changing a nation: The development and evaluation of a training program for oncology health professionals in the provision of genetic testing for ovarian cancer patients
}

Antill $\mathrm{Y}^{1,2,3}$, Meiser $\mathrm{B}^{4}$, Do J ${ }^{4}$, Nevin S, ${ }^{4}$ Gleeson $\mathrm{M}^{5}$, Kentwell $\mathrm{M}^{1}$, Tucker $\mathrm{K}^{6,11}$, Taylor $\mathrm{N}^{7}$, Kirk J8, Barlow-Stewart $\mathrm{K}^{9}$, Friedlander $\mathrm{M}^{10,11}$

${ }^{1}$ Family Cancer Clinic, Royal Melbourne Hospital, ${ }^{2}$ Familial Cancer Clinic, Cabrini Health, ${ }^{3}$ Familial Cancer Clinic Monash Health, ${ }^{4}$ Psychosocial Research Group, Prince of Wales Clinical School, UNSW Sydney; ${ }^{5}$ Hunter Family Cancer Clinic, Newcastle; ${ }^{6}$ Hereditary Cancer Clinic, Prince of Wales Hospital; ${ }^{7}$ The Cancer Council New South Wales; ${ }^{8}$ Familial Cancer Service, Westmead Hospital; ${ }^{9}$ Sydney Medical School, University of Sydney; ${ }^{10}$ Dept Medical Oncology, Prince of Wales Hospital, ${ }^{11}$ Prince of Wales Clinical School, University of New South Wales,

\section{BACKGROUND}

- Approximately $15 \%$ of women with high-grade, nonmucinous, epithelial ovarian/fallopian tube/peritoneal cancer (EOC) carry a germline mutation in BRCA1/2 with up to $40 \%$ of carriers having no significant family history ${ }^{1}$.

- Traditional genetic testing models rely on family history of breast and/or ovarian cancer and require referral to a family cancer clinic (FCC), with referral and testing rates remaining poor in most countries.

- Mainstreaming testing, a model of Genetic testing provided through normal oncology services with FCC follow up as required, has been shown to increase the rate and turn around time for results for EOC women 2,3 .

- We have developed a clinical training initiative to introduce a 'mainstreaming' approach to genetic testing for women with EOC cancer in all cancer centres at an Australasian wide effort.

\section{METHODS}

- A training module was developed for use by core educators for small group sessions.

- The module includes: a presentation describing the rationale behind and suggested approach to mainstream testing in EOC patients including consenting and providing results, a resource folder containing: a suggested flowchart (Fig 1), site specific consent and request forms developed for each site (Fig 2), suggested script or cue sheets for non genetics specialists and patient information brochures about gene testing (Fig 3)

- Non-genetics, oncology healthcare providers were invited to take part in seminars at their local hospital or at local or national educational meetings with participants being provided with a resource folder at the seminar to use in their local site.

- Genetic testing: Copies of the completed forms were sent to both the testing laboratories and the local FCC with testing results given to the patient by local oncology team members.

- FCC referral was arranged when the result was: mutation positive, a variant of uncertain significance or where there was no mutation identified but additional relevant family history of cancers

- All trainees were surveyed at baseline to assess perceived skills, competence and barriers using a validated tool in offering genetic testing to women with EOC and will be surveyed again at 12 months to determine changes

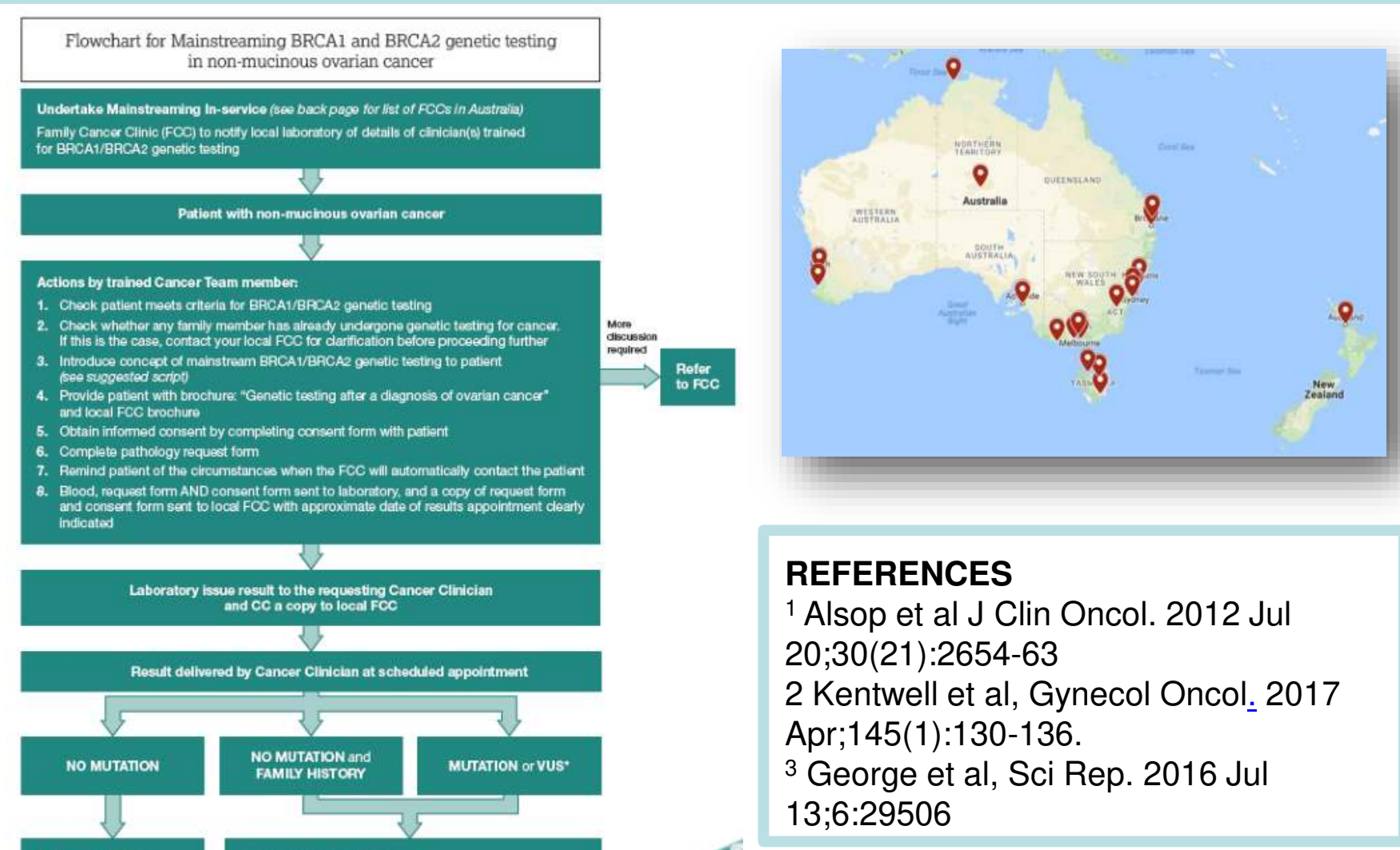

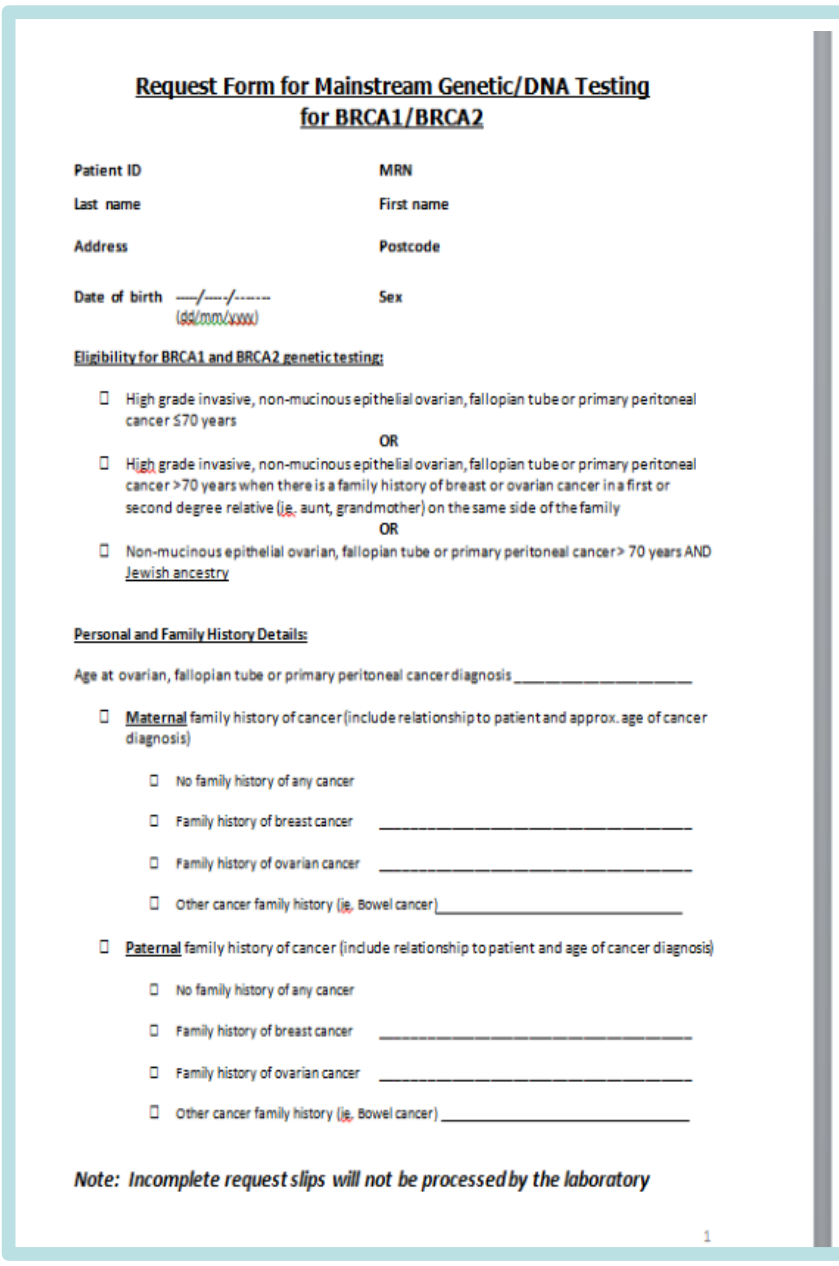

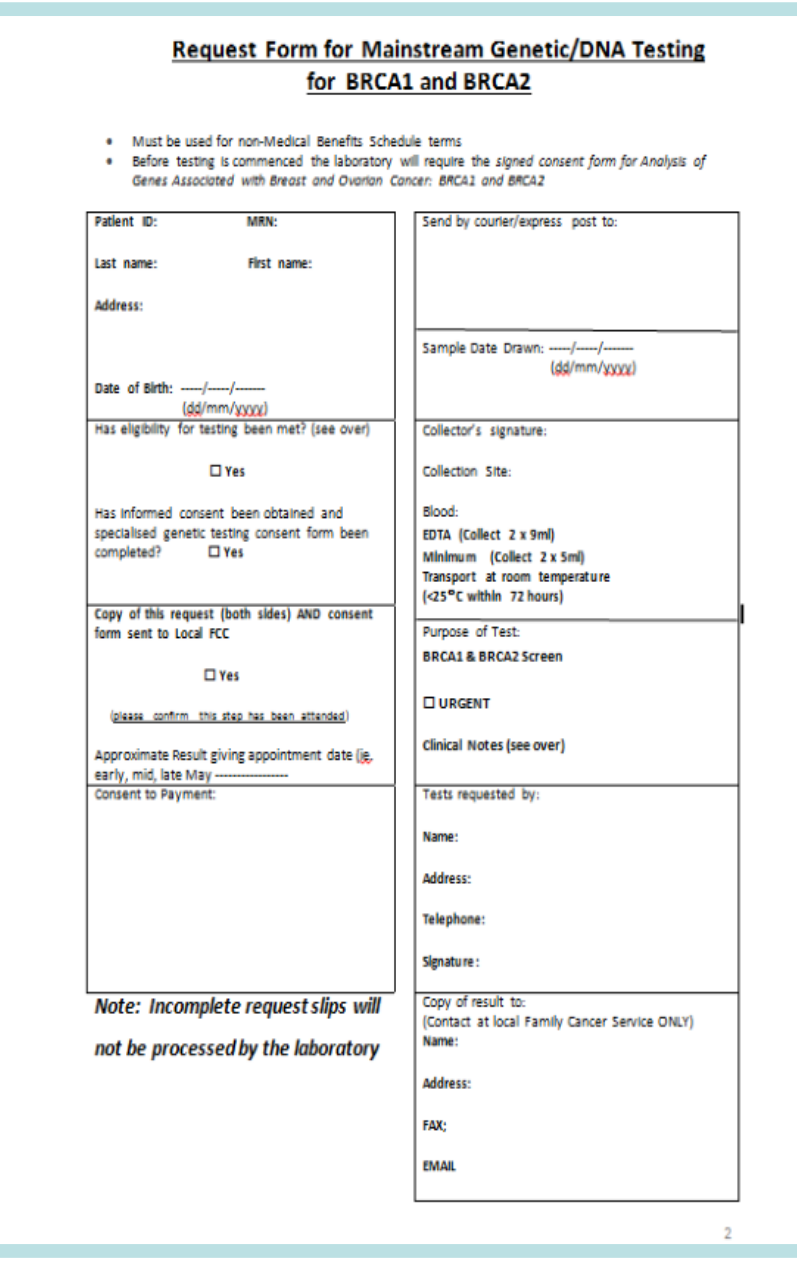

Figure 2
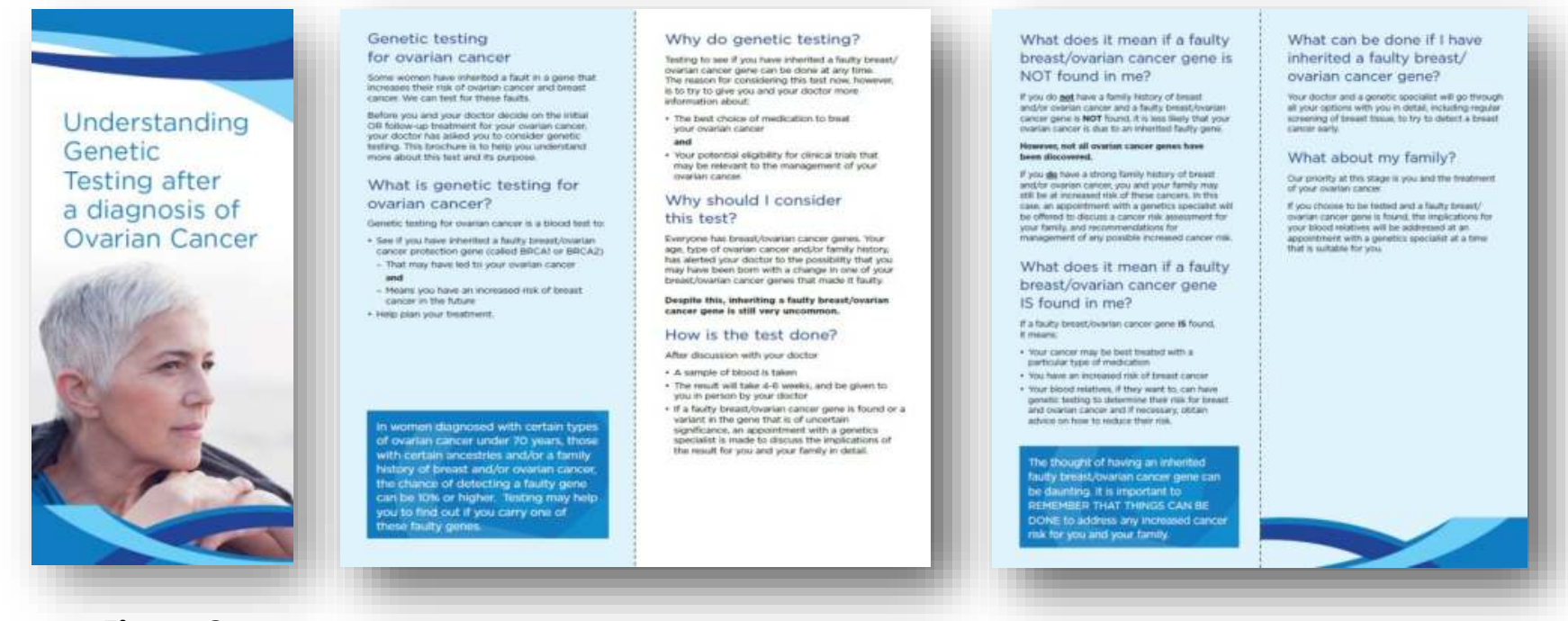

Figure 3

\section{RESULTS}

- All 16 FCCs throughout Australia participated in this clinical and research initiative.

- One hundred and eighty-five oncology health professionals have been trained in 42 workshops.

- Site specific training was carried out in 29 hospitals: 19 of these were within urban areas and 10 from nonurban areas.

- Of these,12 of the 29 hospitals had no onsite FCC.

- Those trained included: 85 (45.9\%) medical oncologists, 27 nurses (14.6\%), 42 (22.7\%) gynae-oncologists, 7 (3.8\%) obstetricians and gynaecologists, and 24 (13\%) 'Other' (researchers, trial or care coordinators, palliative physicians, radiation oncologists, breast surgeons).

- At baseline, 114 (73.1\%) oncology health professionals were aware of national referral and testing guidelines

- Of the oncology health professionals: 47 (30.1\%) 'Always' referring women who meets guidelines for $B R C A 1 / 2$ genetic testing, $51(32.7 \%)$ 'Nearly always', 22 (14.1\%) 'Sometimes', $14(9 \%)$ 'Very little' and 19 (12.2\%) 'Never'.

\section{CONCLUSIONS}

- Non-genetics specialists have demonstrated a willingness to be involved in the provision of genetic testing for selected high-risk groups such as high grade EOC

- This allows wider testing to individuals without the necessity, in most cases, of additional FCC appointments.

- This model is being trialled in other tumour types with ongoing plans for assessment of patient and clinician preference and economic evaluations 